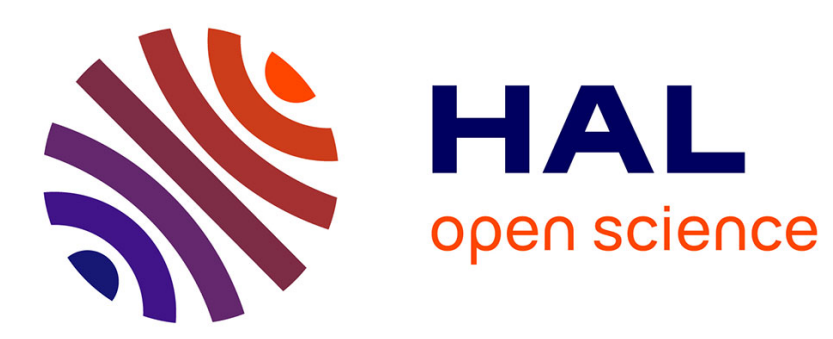

\title{
L'escarpement de faille de la Têt est-il le résultat de la tectonique active Plio-Pléistocène ou d'une exhumation Pléistocène?
}

Jean-Michel Carozza, Stéphane Baize

\section{- To cite this version:}

Jean-Michel Carozza, Stéphane Baize. L'escarpement de faille de la Têt est-il le résultat de la tectonique active Plio-Pléistocène ou d'une exhumation Pléistocène?. Comptes Rendus Géoscience, 2004, 336, pp.217-226. halshs-01066287

\section{HAL Id: halshs-01066287 \\ https://shs.hal.science/halshs-01066287}

Submitted on 19 Sep 2014

HAL is a multi-disciplinary open access archive for the deposit and dissemination of scientific research documents, whether they are published or not. The documents may come from teaching and research institutions in France or abroad, or from public or private research centers.
L'archive ouverte pluridisciplinaire HAL, est destinée au dépôt et à la diffusion de documents scientifiques de niveau recherche, publiés ou non, émanant des établissements d'enseignement et de recherche français ou étrangers, des laboratoires publics ou privés. 
Tectonics

\title{
L'escarpement de faille de la Têt est-il le résultat de la tectonique active Plio-Pléistocène ou d'une exhumation Pléistocène?
}

\author{
Jean-Michel Carozza $^{\mathrm{a}, *}$, Stéphane Baize ${ }^{\mathrm{b}}$ \\ a Département de géographie, université Louis-Pasteur, 3, rue de l'Argonne, 67083 Strasbourg cedex, et GEODE, UMR 5602 CNRS, France \\ ${ }^{\mathrm{b}}$ Institut de radioprotection et de sûreté nucléaire, 18, route du Panorama, 92260 Fontenay-aux-Roses, France
}

Reçu le 25 mai 2003 ; accepté après révision le 28 octobre 2003

\begin{abstract}
Résumé
L'expression morphologique de la faille de la Têt ressemble à celle d'une faille active. Son fonctionnement, attesté pour le Pliocène, reste cependant débattu pour le Pléistocène. Un schéma d'évolution de l'escarpement de faille est proposé. Il se base sur les relations entre morphologie de l'escarpement et remplissage sédimentaire des bassins. Il souligne l'importance des phénomènes d'exhumation par incision du réseau hydrographique au cours du Pléistocène. Ainsi, les mouvements tectoniques pléistocènes apparaissent faibles et leur impact sur les caractéristiques du drainage limité. Le schéma proposé conduit également à minorer le rejet au cours du Pliocène, celui-ci étant réévalué entre 150 et $300 \mathrm{~m}$. Une grande partie des mouvements tectoniques responsables du relief actuel sont donc antérieurs. Pour citer cet article : J.-M. Carozza, S. Baize, C. R. Geoscience 336 (2004). ๑ 2004 Académie des sciences. Publié par Elsevier SAS. Tous droits réservés.
\end{abstract}

\section{Abstract}

Is the Fault Têt the result of Plio-Pleistocene tectonic activity or Pleistocene exhumation? The Têt Fault looks like an active fault. Its activity, attested for Pliocene, remains discussed for Pleistocene. We propose a new scenario of evolution for the Têt Fault, highlighting the importance of Pleistocene exhumation processes. It is based on a relationship between morphology and basin filling. During Pleistocene, the fault activity is weak and vertical displacements seem to have no considerable impact on the drainage features. This study concludes that Pliocene offset may also be reduced: the fault activity generating relief is anterior to Pliocene. To cite this article: J.-M. Carozza, S. Baize, C. R. Geoscience 336 (2004).

C 2004 Académie des sciences. Publié par Elsevier SAS. Tous droits réservés.

Mots-clés : Pyrénées orientales ; faille ; exhumation ; néotectonique ; incision ; France

Keywords: Eastern Pyrenees; fault; exhumation process; neotectonics; downcutting; France

\footnotetext{
* Autheur correspondant.

Adresse e-mail : carozza@geographie.u-strasbg.fr (J.-M. Carozza).

1631-0713/\$ - see front matter @ 2004 Académie des sciences. Publié par Elsevier SAS. Tous droits réservés. doi:10.1016/j.crte.2003.10.026
} 


\section{Abridged English version}

\section{Introduction}

The eastern termination of the Pyrenean orogenic belt presents a moderate seismotectonic activity, but with significant instrumental record (Fig. 1a) [16,20, 23]. The major seismic crisis of the area occurred during 1427 and $1428(M l=6.1)$, but fault source(s) is (are) not identified, neither with surface rupture nor with macroseismic mapping (Amer fault or Vallfogona Thrust, Têt Fault?). On a general way, seismic events are not linked with major geomorphic structure in eastern Pyrenees. Nevertheless, the Têt Fault was assumed to be the source of the seismic crisis of the 1427-1428 seismic events, mainly according to morphological evidences [4,7]. This fault is $80 \mathrm{~km}$ long, trending NE-SW. It was an active fault during Oligo-Miocene times [6], inducing an important deposition of marine and continental sediments. The current scarp associated with the fault, between the basement to the south and the Cainozoic basin to the north, shows actually some characteristic shapes that can be interpreted as an active fault features [4,7]: sharp contact between hangingwall and footwall, low sinuosity front index (less than 1.4), triangular spur morphology ... (Figs. 1b and 2). However, no evidence for Pleistocene activity was referred along it. The aim of this paper is to point out geomorphic evidences for a partial exhumation origin of this fault during Pleistocene time.

\section{Scarp morphology and relationship to basin filling}

In order to assess the Plio-Pleistocene offset of the fault, we use geomorphologic indirect data, in addition with field investigations. Longitudinal profiles of tributaries of the Têt River, cutting perpendicularly the fault trend, reveal different morphological pattern along the fault. In the eastern part, the typical profile is described by an exponential function, whereas a linear function fits the data in the western part. This longitudinal differentiation of drainage and relief pattern along the fault was first interpreted as a westward propagation of uplift due to Plio-Pleistocene active faulting $[8,10]$. The main argument was that the first class of profiles corresponds to relative immature drainage basins, whereas the second corresponds to relative mature basins, which is in a good agreement with the hypsometric curves [10].

New results recently obtained enable to refine the tectonic events' chronology.

Detailed analyses of the tributaries longitudinal profiles show systematic knickpoints located around $4 \mathrm{~km}$ behind the fault trace (Fig. 3). These 'perched valley' morphologies (i.e., the upstream part of the profile) were previously considered to be tectonic related, and were supposed to attest the uplift of the footwall due to normal faulting of the Têt Fault during Pleistocene time [4,7]. However, these knickpoints can also reflect down cutting associated with local base level change [5]. We computed longitudinal profiles of the main river before erosion by best-fitting calculated equation of the upstream part of the profile beyond the knickpoint. This fitting excludes the uppermost segment of the profile, where glacial or periglacial processes affected drastically the morphogenesis. In a geomorphological point of view, the knickpoint behaves as a local base level for this uppermost segment, which is not affected by downstream evolution. In the Canigou area, the limit of glacial removing is $1400 \mathrm{~m}$.

The computed profiles for nine tributaries crossing the fault give an average vertical incision of $120 \mathrm{~m}$, reaching to more than $300 \mathrm{~m}$ (Fig. 3). Lower Pleistocene terrace of the Llech Basin is located $120 \mathrm{~m}$ above current talweg downward from the knickpoint. An important conclusion is that knickpoint is younger than the Lower Pleistocene. In addition, downward continuation of the computed profile is in perfect link with the summit of the Conflent sedimentary filling, which is now considered as Pliocene [8,13]. Stages of the incision can be partially reconstituted according to alluvial terrace chronology available in the Llech Basin. Three generations of strath terraces are attributed, from the highest to the lowest relative to current talweg, to Middle Pleistocene $(+120 \mathrm{~m})$, Upper Pleistocene $(+50 \mathrm{~m})$ and Late Glacial $(+15 \mathrm{~m})$. According to the data, completed with investigation on other basins, the following scenario of the basin history and river incision can be proposed.

(1) During Lower to Median Pliocene, aggradation of syntectonic alluvial deposits fills the Conflent and the Roussillon Basin.

(2) The 'summit-level surface' of the Pliocene alluvial fans marks the initial stage of Pleistocene 
incision. Relict evidences of this level are currently identified as erosional surfaces in the Conflent Basin or preserved deposits (the 'Col de Fins' formation, Fig. 4(b) [7]). It maintained since Late Pliocene or Early Pleistocene.

(3) Later, in response to sea level falling, degradation and exhumation are initiated. The half part of the exhumation occurred during Lower Pleistocene. The last glacial cycle represents only ten per cent of the total exhumation.

\section{Discussion and conclusion}

Preceding remarks suggest that incision is the main process of the morphogenesis of present-day topography. The scarp morphology previously interpreted as tectonic triangular spurs, corresponds largely to an exhumed feature: the buried fault plane (under Pliocene) is exposed after differential erosion affected easily soft Pliocene sediments and preserved bedrock on the footwall. By reconstructing the alluvial filling of the Conflent Basin, it appears that the two third of the height of the cumulative scarp may be due to the exhumation of the fault. For example, in the Thuès-les-Bains area, in the western part of the Conflent Basin, near Thuès), the Têt Fault exhibits a 600 m-high spur. But only 200 m of this scarp can be associated to active faulting. Thus, the importance of Plio-Pleistocene uplift in relief genesis must be reassessed in eastern Pyrenees.

Contrary to the previous model (e.g., [7]), we suggest that the main relief is acquired as soon as the Middle Miocene. According to geological evidences, the major normal faulting may have occurred before the end of Miocene. Our geomorphologic results, consistent with new apatite fission trace data [18], indicate an early genesis of the Canigou horst (Upper Oligocene: 27-26 Myr), in relation with the Gulf of Lion rifting stage. The major tectonic event in eastern Pyrenees caused a coeval hydrographical incision, which is recorded by the lower syntectonic series [3]. The vertical offset would be around $2000 \mathrm{~m}$ [21].

After this Messinian event, an ultimate significative tectonic event is recorded by the Escaro syntectonic deposits, which implied 200 to $300 \mathrm{~m}$ vertical offset along the Têt Fault. The data developed before suggest that the Late-Pliocene-to-Early-Pleistocene evolution is mainly characterized by the hydrographic incision superimposed upon the top of the Mio-Pliocene allu- vial filling, which may be responsible of the exhumation of the scarp. We propose, according to this new interpretation of geomorphologic data and new dating of alluvial deposits, that Pleistocene vertical offset must be reduced. Indeed, the fault is sealed at least in two sites by non-deformed alluvial fans attributed to Middle Pleistocene. This implies weak to null vertical offset of the Têt Fault since Middle Pleistocene.

\section{Introduction}

La terminaison orientale de la chaîne pyrénéenne est un domaine d'activité sismo-tectonique modérée, mais significative d'un point de vue instrumental [20, 23]. Cette sismicité apparaît plutôt diffuse et difficile à rattacher à des failles reconnues en surface (Fig. 1a). De la même manière, le lien entre les séismes de 14271428 et une structure sismogène a fait l'objet de différentes hypothèses : chevauchement de Vallfogona [21]; faille normale de l'Amer [15]; faille normale de la Têt [4]. Cette dernière hypothèse se fonde sur l'existence, dans la région, de failles (failles de la Têt, du Tech, du Capcir) dont l'expression morphologique rappelle celle des failles actives : contact tectonique non dégradé entre le socle et le remplissage détritique meuble des bassins, tracé rectiligne (indice de sinuosité du front inférieur à 1,4), présence de facettes triangulaires régulières et marquées dans le paysage (Figs. 1b et 2). Ce type de morphologie est particulièrement visible sur la faille de la Têt. Cette dernière, de direction NE-SW, est longue d'environ $80 \mathrm{~km}$. Elle met en contact les terrains granito-gneissiques du massif hercynien du Canigou, au sud, et les séries sédimentaires néogènes, au nord. Cette faille a fonctionné au cours de l'Oligo-Miocène et du Pliocène [6, 8]. Trois générations de facettes imbriquées, correspondant à des phases d'activité de la faille, ont été identifiées (Fig. 2). Des considérations principalement géométriques discutées plus loin ont conduit certains auteurs à relier la dernière génération de facettes à une activité pléistocène s.1. [2,7]. Pour les périodes récentes, il n'existe cependant aucune preuve directe de l'activité de cette faille; en particulier, aucun indice de rupture affectant le Pléistocène n'a été décrit en association directe avec l'escarpement [1]. S'il est vrai que la morphologie de cet escarpement est très proche de celle d'une faille active, la présence de facettes ne constitue pas à elle seule un critère suffisant pour dé- 


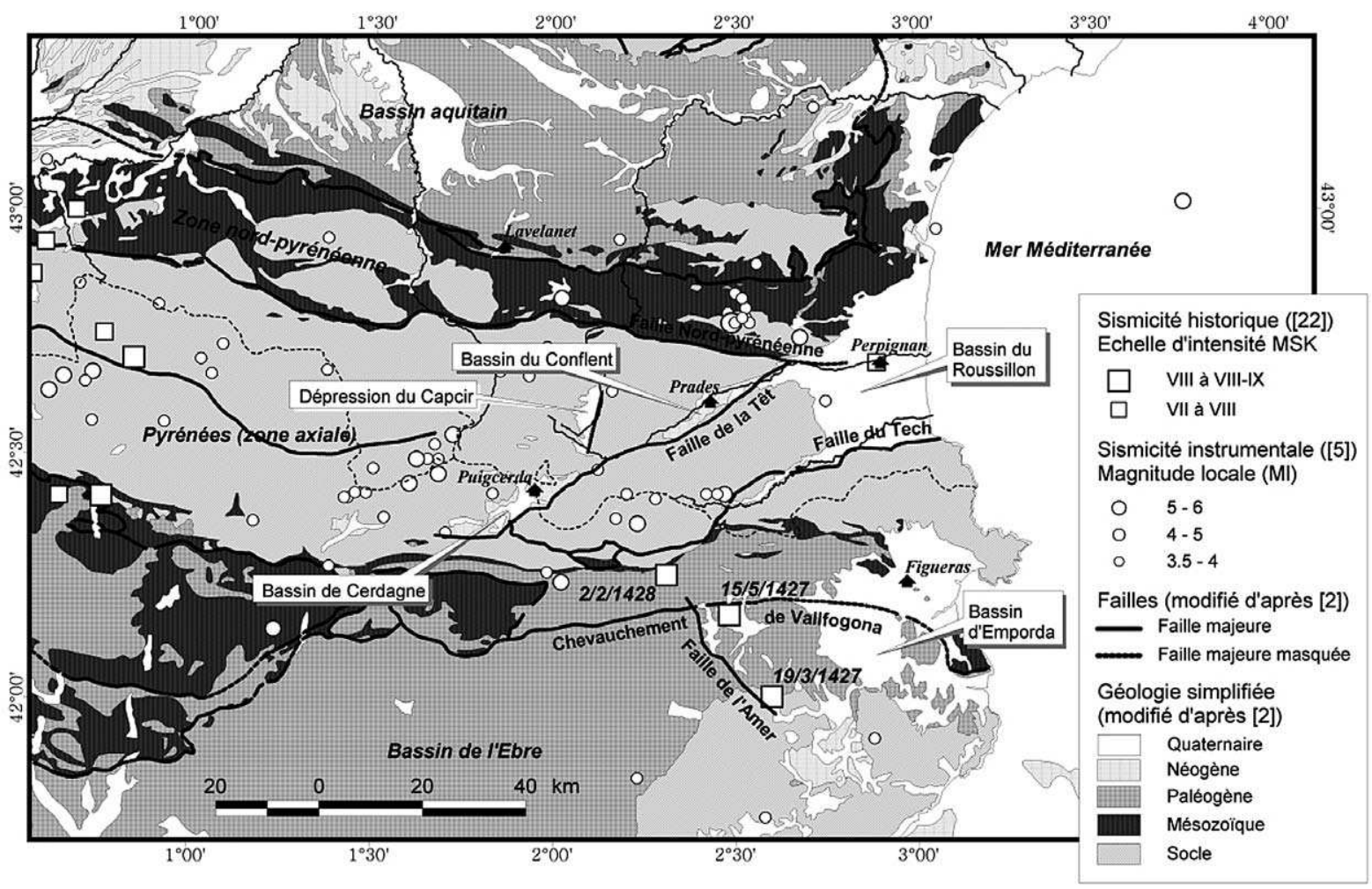

(A)

Fig. 1. (A) Carte géologique simplifiée des Pyrénées orientales (modifiée d'après [3]). La sismicité historique est représentée par les épicentres des événements principaux. La sismicité instrumentale est extraite du catalogue CEA/LDG [12]. (B) Modèle numérique de terrain (MNT) ombré de la région de la faille de la Têt (d'après le MNT de l'Institut géographique national, maille de $50 \mathrm{~m}$ ). Le tracé des failles représentées est modifié d'après [3]. Cette figure illustre la morphologie à facettes de l'escarpement associé à la faille de la Têt.

Fig. 1. (A) Simplified geological map of the Eastern Pyrenees (modified from [3]). Historical seismicity is represented by epicentres locations of main events. Current seismicity is extracted from the CEA/LDG database [12]. (B) Shaded Digital Elevation Model (DEM) of the surrounding area of the Têt Fault (DEM from the French National Geographic Institute, grid size: $50 \mathrm{~m}$ ). The fault traces are modified from [3]. This picture shows the spur morphology of the cumulative scarp associated with the Têt Fault.

montrer une origine tectonique [4]. En effet, les facettes peuvent résulter d'un processus d'exhumation, qui aurait pour effet de majorer le jeu apparent de la faille.

Dans ce travail, nous discutons, à partir de considérations géomorphologiques et morphométriques, l'origine de l'escarpement de faille de la Têt et de la dernière génération de facettes associées, les plus récentes (facettes d'ordre 1), qui ont servi d'argument pour attester une activité en faille normale de la faille de la Têt au cours du Pléistocène [4]. Nous déduisons de ce travail une ré-évaluation du rôle de la néo-tectonique dans la mise en place du relief actuel des Pyrénées orientales au cours du Plio-Pléistocène.

\section{Morphologie du front et relation avec le remplissage du bassin}

L'étude des relations entre le front orographique, i.e., l'escarpement cumulé associé à la faille de la Têt, et le remplissage sédimentaire a été menée suivant une approche qui combine travaux de terrain et détermination de paramètres morphométriques du drainage, en particulier la morphologie des profils en long des thalwegs.

Les profils en long des cours d'eau drainant le front sud du massif du Canigou présentent une forte variation de leur géométrie. Un gradient d'organisation est mis en évidence par une transition progressive du type 


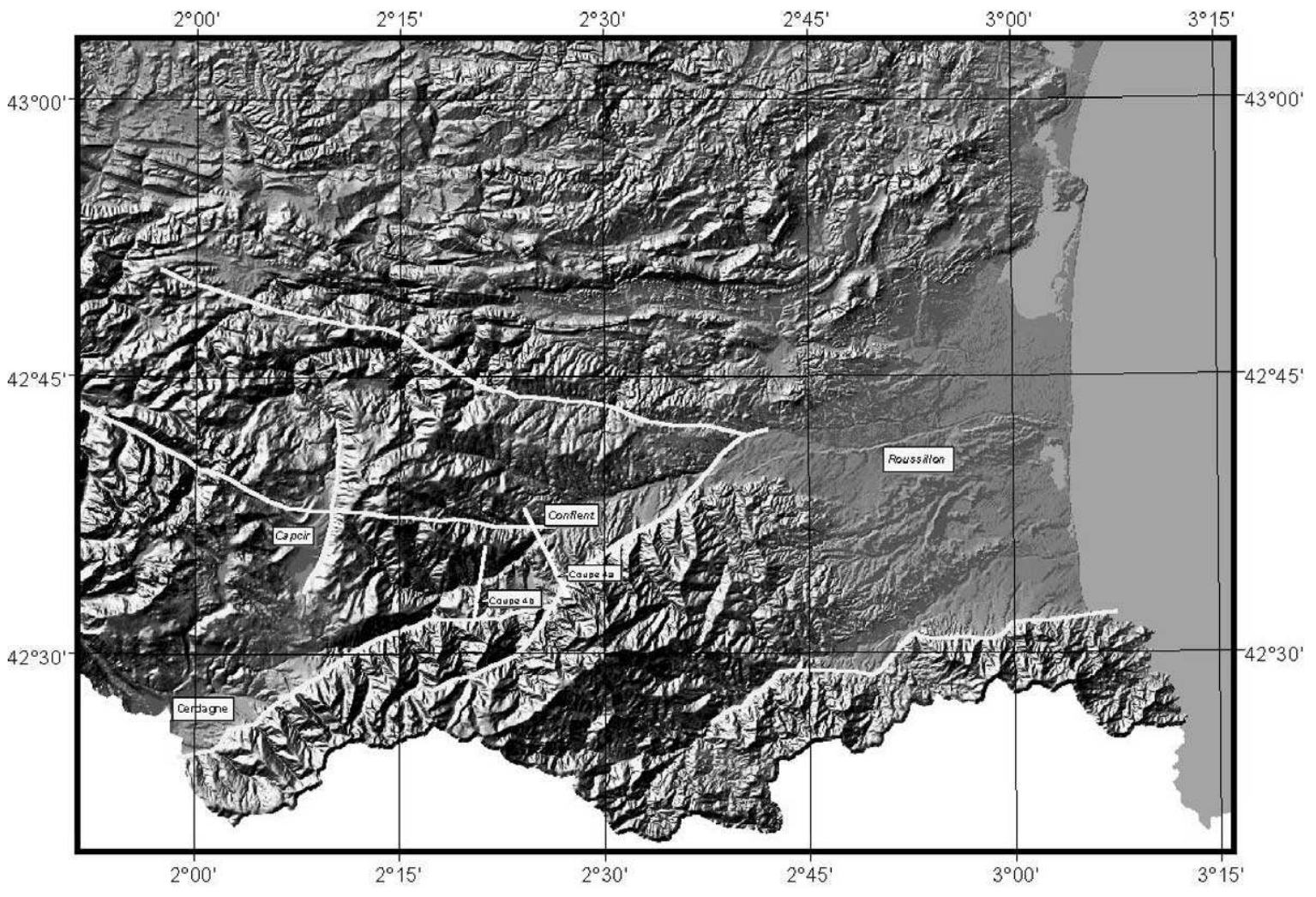

(B)

Fig. 1. (Continued).

d'équation décrivant les profils longitudinaux. Ceuxci sont de type exponentiel et très régulier à l'est; ils passent à des profils de type linéaire à l'ouest. Cette organisation a, dans un premier temps, été interprétée comme la réponse des bassins versants à un gradient de surrection du massif du Canigou au cours du Plio-Pléistocène, avec une propagation de la surrection vers l'ouest $[8,10]$. De nouvelles observations réalisées depuis permettent d'affiner la chronologie des événements ayant généré le relief de cette partie de la chaîne et de relier ceux-ci aux principaux événements géodynamiques régionaux.

L'analyse détaillée de ces profils en long montre la présence systématique de ruptures de pente, qui segmentent en deux parties les profils. Ces morphologies correspondent à des knickpoints [5]. Un exemple de ce type de morphologie est donné sur la Fig. 3. Ces ruptures sont fréquemment associées à des systèmes de gorges incisant le socle. Elles se positionnent en moyenne à 4,2 km en arrière du franchissement de la faille. Elles ont été précédemment interprétées comme la preuve de l'activité récente de la faille [4]. Dans ce schéma, la déformation cumulée le long de la faille aurait induit la surrection du horst du Canigou et la mise en place d'une vague d'érosion régressive au niveau des talwegs, enregistrée par les knickpoints. Ces derniers auraient une origine tectonique, déterminant une morphologie en «vallées suspendues », signant un fonctionnement en faille normale de l'accident de la Têt [4]. L'inachèvement de la régularisation du profil en long serait alors une preuve du caractère «récent» de la déformation le long de la faille. Chronologiquement, ils ont été attribués au Pléistocène s.l. Cependant, d'autres processus peuvent être responsables de la formation de knickpoints, ces derniers constituant une réponse des cours d'eau à une modification du niveau de base, qui peut être d'origine tectonique, eustatique (c'est-à-dire liée au niveau de base) ou à la dy- 


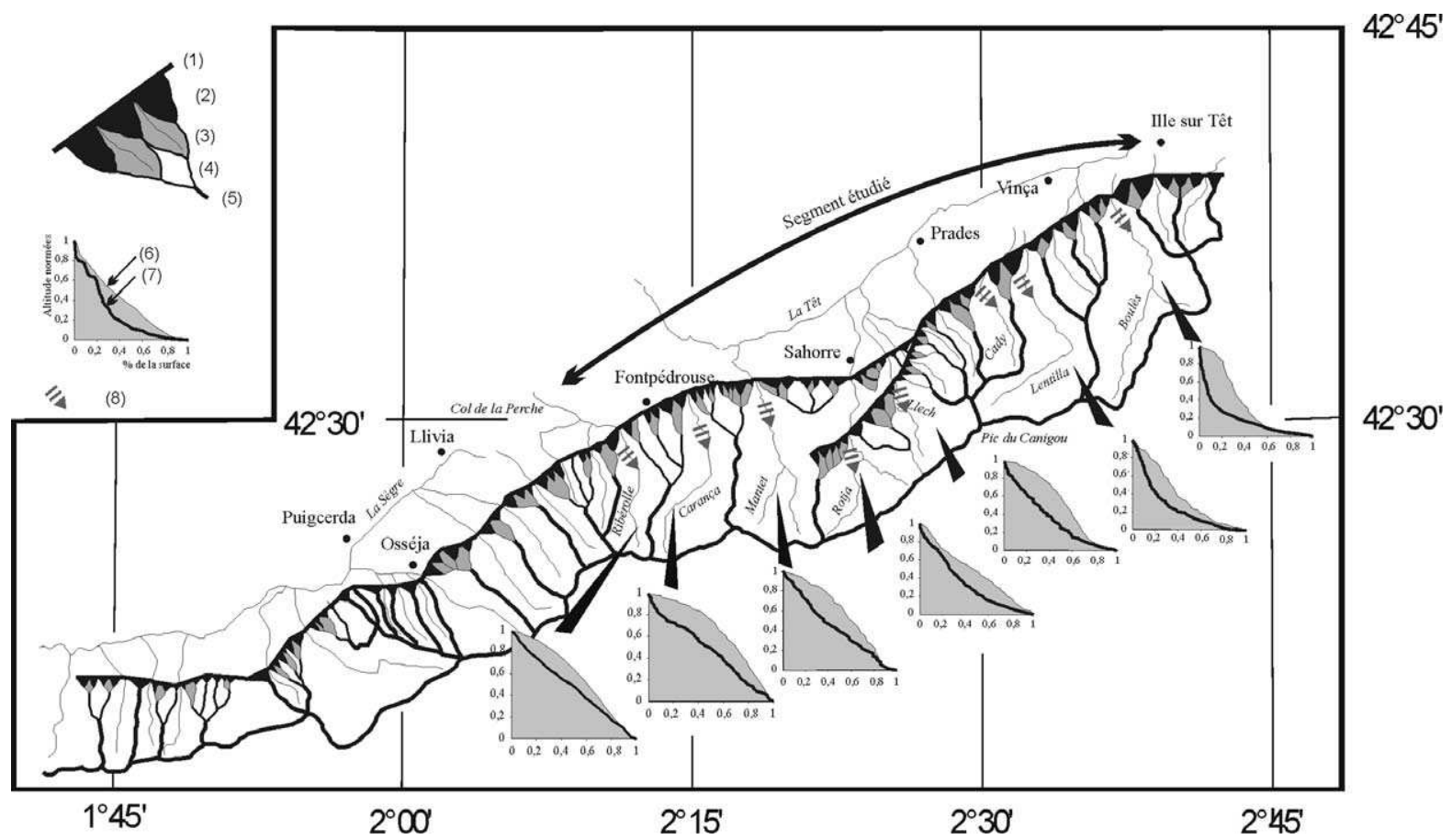

Fig. 2. Morphologie de l'escarpement associé à la faille de la Têt, avec les différentes générations de facettes triangulaires. Les principaux cours d'eau référencés dans le texte sont localisés. 1. Faille principale 2. Facette d'ordre 1. 3. Facettes d'ordre 2. 4. Facette d'ordre 3. 5. Ligne de crête principale.

Fig. 2. The Têt Fault scarp morphology, with the three spur generations. The main rivers cited in the text are localized in this picture. 1. Main fault 2. First-order spur. 3. Second-order spur 4. Third-order spur. 5. Main crest line.

namique propre du système fluvial, notamment sous contrôle climatique.

Nous avons procédé, dans un premier temps, au calcul de l'équation décrivant les profils longitudinaux des cours d'eau sur le segment en amont du knickpoint, dans le compartiment soulevé (c'est-à-dire le socle du massif du Canigou) pour l'ensemble des huit cours d'eau principaux. Puis nous avons généralisé l'équation obtenue vers l'aval (c'est-à-dire vers le bassin) pour restituer le profil du cours d'eau avant la mise en place du processus d'érosion régressive à l'origine du knickpoint. Cette technique, purement descriptive, permet de s'affranchir du problème de l'origine des knickpoints. Cette démarche apparaît validée par le fait que le knickpoint se conduit comme un niveau de base local pour le segment amont du bassin versant. Celui-ci échappe ainsi en grande partie à l'impact des modifications du niveau de base et conserve son profil en long. Dans le domaine étudié, l'influence de la morphogenèse glaciaire est importante dans l'amont des bassins versants. Ceci conduit à écarter du calcul la partie la plus élevée du profil, en l'occurrence les altitudes au-delà de $1400 \mathrm{~m}$, valeur qui est reconnue comme la limite inférieure de l'englacement dans le massif du Canigou [7,17]. Seul un tronçon intermédiaire, compris entre le knickpoint et la limite aval de l'action de glacier, a donc été pris en considération. Diverses fonctions mathématiques simples d'ajustement entre altitude et distance ont été testées pour chacun des profils (fonctions linéaire, exponentielle ou polynomiale d'ordre 2) et les meilleurs ajustements retenus. Pour le profil présenté ici à titre d'exemple (Fig. 3), le meilleur ajustement est obtenu pour une fonction exponentielle, et le coefficient de détermination est excellent $\left(r^{2}=0,99\right)$. Les profils reconstitués pour les huit bassins versants aboutissent à une évaluation de l'incision verticale du talweg, autrement dit la différence d'altitude entre le talweg actuel et le talweg reconstitué, de $120 \mathrm{~m}$ en moyenne. Cette valeur est cependant très variable et s'accroît vers l'est, où 


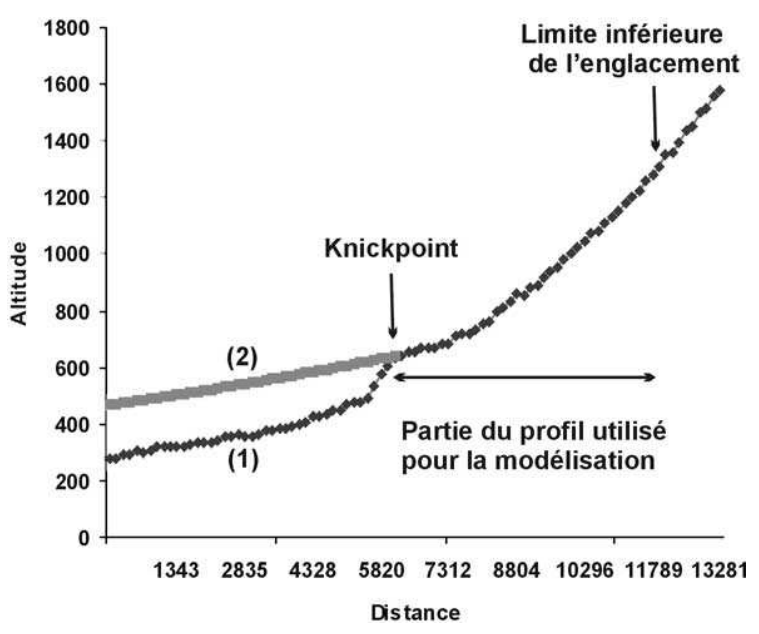

Fig. 3. Exemple de reconstitution du profil longitudinal du Llech. 1. Profil extrait du modèle numérique de terrain. 2. Profil reconstitué par généralisation de l'équation d'amont. Exagération verticale : $\times 6$.

Fig. 3. Example of computed profile of the Llech River. 1. Profile extracted from Digital Elevation Model. 2. Computed profile. Vertical exageration: $\times 6$.

elle peut atteindre plus de $300 \mathrm{~m}$. Les valeurs obtenues sont cohérentes avec les dispositifs géométriques des terrasses étagées, qui permettent de reconstituer les étapes de l'incision des cours d'eau.

À l'aval de la faille, le profil reconstitué se raccorde au sommet du remplissage du bassin du Conflent (formation d'Escaro), dont il constitue le prolongement géométrique (Fig. 4a). Cette formation détritique, anciennement attribuée à l'Oligocène [2], a récemment fait l'objet d'une révision stratigraphique et est aujourd'hui rapportée au Pliocène ancien à moyen sur la base d'arguments géométriques [14] et sédimentologiques [8]. La partie du profil du Llech localisée en amont du knickpoint a donc peu évolué depuis le début du Pléistocène et les volumes, indépendamment des retouches par les glaciers, ont été peu modifiés.

Un scénario d'incision des cours d'eau drainant le flanc nord du massif du Canigou peut être envisagé comme suit. Au cours du Pliocène ancien à moyen, le dépôt des séries syntectoniques d'Escaro (sommet du remplissage du bassin du Conflent) s'achève par la mise en place d'un ensemble de cônes coalescents formant une surface d'abandon. Elle est équivalente à celle définie en Roussillon [13]. La géométrie de cette forme est encore aujourd'hui partiellement conservée sur certaines des interfluves. La spécificité sédimentologique (intervalle condensé) permet également sa reconnaissance (formation dite du Col de Fins [7]). La surface d'abandon reste fonctionnelle jusqu'au Pliocène supérieur, bien qu'elle soit peut-être déjà partiellement démantelée par l'incision fluviale au cours du bas niveau eustatique du cycle TB 3-5 et 3-6 (3,8 et 2,9 Ma) [17]. L'incision provoquée à ce stade reste toutefois minime, puisqu'en Roussillon, la très haute terrasse T5 de même âge n'est pas incisée dans le remplissage pliocène. À partir de cette surface d'abandon des cônes fini-pliocènes, la Têt a incisé son substratum (remplissage mio-pliocène ou, localement, socle hercynien) en suivant un tracé minimisant la distance au niveau de base. De plus, il est probable que la pente de ces cônes issus du massif du Canigou a été suffisamment forte pour bloquer la migration de la Têt vers le sud. La Têt a ainsi constitué les épigénies de Villefranche-de-Conflent et de Vinça par surimposition à partir de la surface d'abandon des cônes pliocènes. Ceci est particulièrement manifeste à la confluence de la Rotja et de la Têt (Fig. 4b). Au final, la reconstitution des quelques profils de terrasses alluviales disponibles permet de quantifier les rythmes d'incision. Les étapes de cette incision peuvent être, en particulier, déduites des données issues de l'étagement des terrasses dans les bassins du Llech et de la Rotja (Fig. 4). Sur ce dernier cours d'eau, trois générations de terrasses à dispositif étagé ont été identifiées. Elles sont attribuées au Pléistocène moyen, au Pléistocène récent et au Tardi-Glaciaire [17]. Elles se localisent respectivement à 120,50 et $15 \mathrm{~m}$ au-dessus $\mathrm{du}$ talweg actuel. Compte tenu de la position initiale du réseau hydrographique tel que nous l'avons restitué, un tiers de l'incision $(80 \mathrm{~m})$ peut être attribué au Pléistocène ancien. Cette valeur correspond à la différence entre la surface reconstituée du cône et l'altitude actuelle de la terrasse la plus ancienne. Les deux tiers restant $(150 \mathrm{~m})$ semblent se répartir également entre le Pléistocène moyen et final. L'incision tardi-glaciaire et holocène ne couvre qu'un dixième de l'incision totale.

\section{Implication pour l'âge des reliefs des Pyrénées orientales}

Pour des raisons de prolongement géométrique de la surface d'abandon des cônes pliocènes vers 


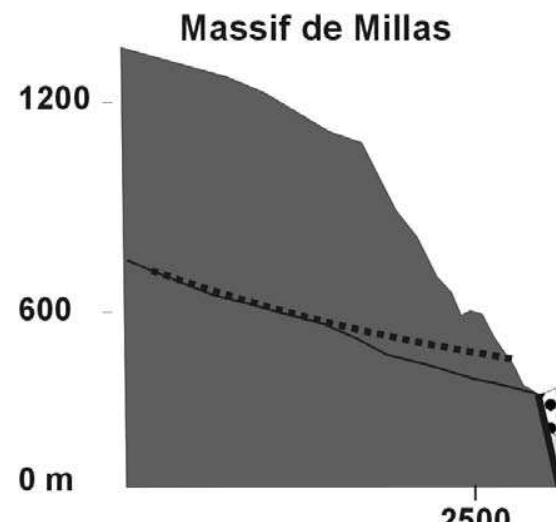

2500

Faille de Prades

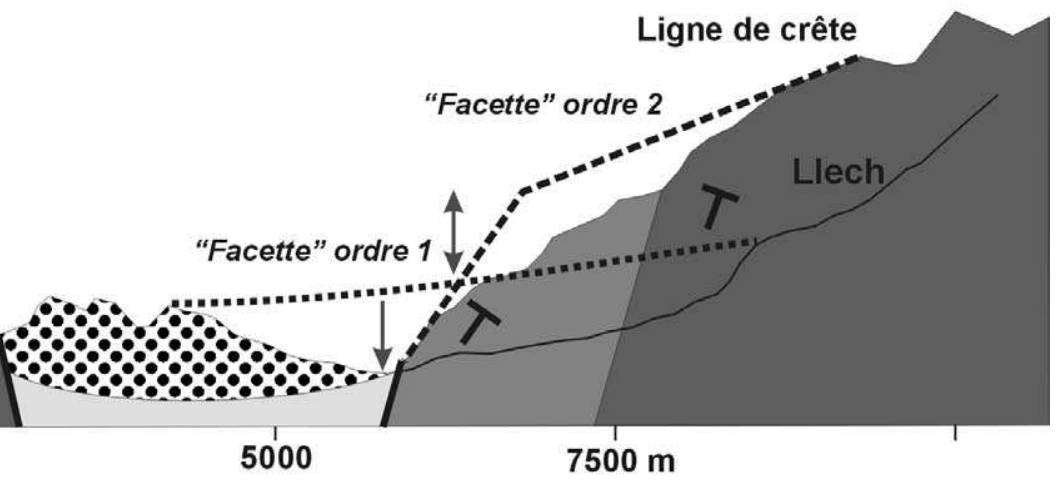

Faille de la Têt

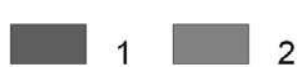

2

$\mathrm{S}$

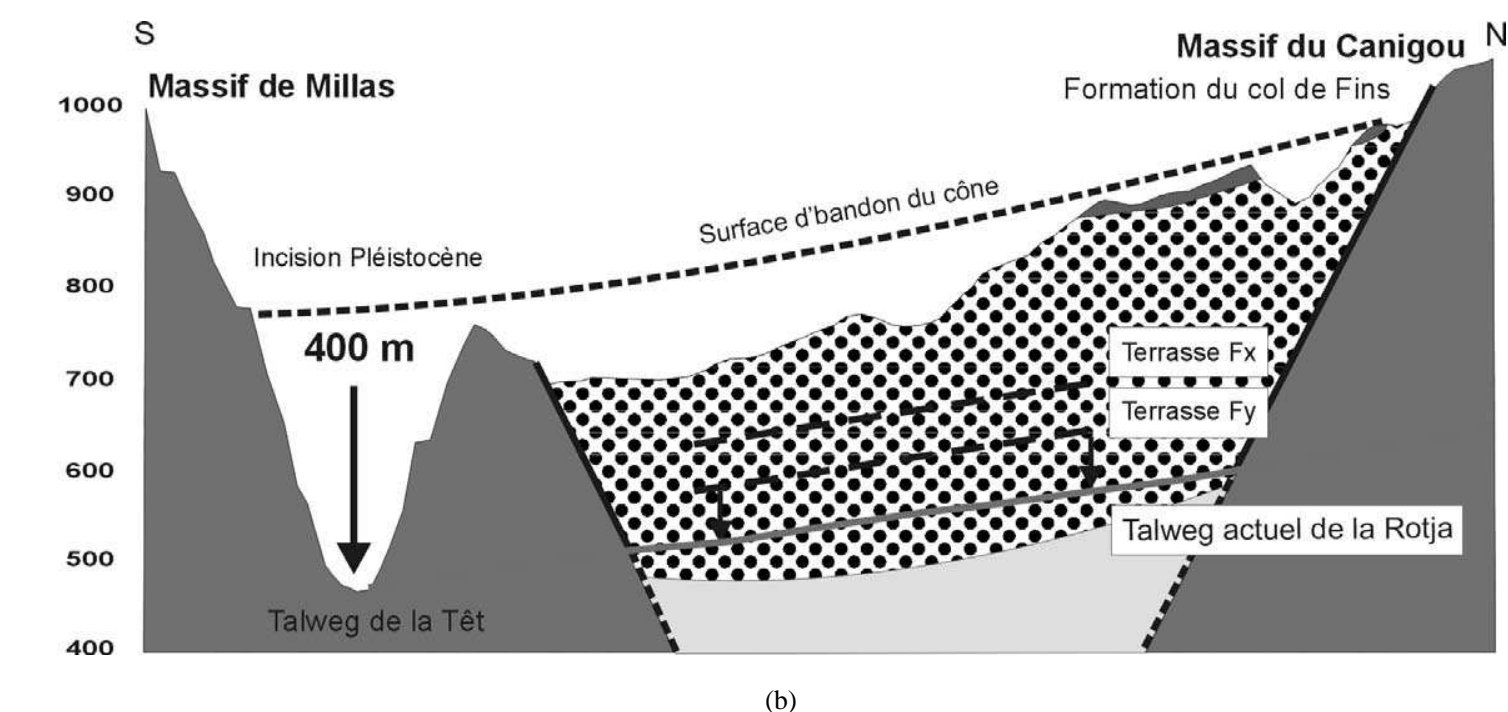

3

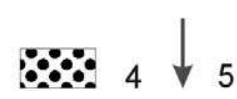

(a)

\section{Massif du Canigou}

\section{igne de crête}

Fig. 4. Relations géométriques et stratigraphiques entre les morphologies des profils en long et le remplissage pliocène du bassin du Conflent. (a) Au niveau de Prades; (b) au niveau de Villefranche-de-Conflent (remarquer l'épigénie de la Têt sur le massif de Millas). 1. Granite et gneiss hercynien. 2. Schistes paléozoïques. 3. Miocène inférieur (Aquitano-Burdigalien). 4. Pliocène. 5. Incision pléistocène. 6. Rejet vertical pliocène. 7. Profil en long restitué. 8. Foliation des gneiss. Fx : Moyenne terrasse d'âge supposé «Mindel». Fy : Basse terrasse d'âge supposé «Riss ».

Fig. 4. Stratigraphic and geometric relationships between morphologies and Pliocene sedimentary infill of the Conflent Basin. (a) In the Prades area. (b) In the Villefranche-de-Conflent area. Note the epigenic position of the Têt River. 1. Hercynian granite and gneiss. 2. Palaeozoic metasediment. 3. Aquitano-Burdigalian. 4. Pliocene. 5. Pleistocene incision. 6. Pliocene offset. 7. Computed longitudinal profile. 8. Gneiss foliation. Fx: Strath terraces 'Mindel'. Fy: Strath terraces 'Riss'.

l'amont du bassin de la Têt, les remarques précédentes conduisent à évaluer une incision maximale de l'ordre de $400 \mathrm{~m}$ au niveau de la gorge de Villefranche-
de-Conflent durant le même intervalle chronologique [11]. Si l'incision progressive de la Têt elle-même est mal documentée faute de niveaux alluviaux intermé- 
diaires conservés, notamment dans le secteur en amont de Villefranche-de-Conflent, il est toutefois possible de la reconstituer par généralisation des données collectées sur ses affluents (Fig. 4b). Nous sommes donc amenés à proposer un rôle de premier ordre pour l'incision et à minorer l'impact de la néotectonique comme facteur de la morphogenèse de la forme actuelle de l'escarpement de faille de la Têt.

Ainsi, la génération de facettes F1, les plus récentes, ressort pour partie d'un processus d'exhumation, variable spatialement entre 200 et $400 \mathrm{~m}$. Dans les gorges de Thuès-les-Bains, où les facettes F1 peuvent atteindre $600 \mathrm{~m}$ [4], l'abaissement du niveau de la Têt en relation avec les modifications du niveau de base est à lui seul responsable d'environ $400 \mathrm{~m}$ de façonnement de ces facettes. Seuls $200 \mathrm{~m}$ peuvent être attribués à l'activité tectonique Plio-Pléistocène. Le façonnement de ces facettes a de surcrô̂t bénéficié de conditions structurales particulières : fort contraste lithologique dans le secteur du bassin entre le socle et le remplissage sédimentaire, positionnement de la foliation des gneiss parallèlement au versant constituant une armature en chevrons pour la retombée du dôme du Canigou. Ces conditions ont permis la conservation des formes.

Ainsi, le rôle de la néotectonique Plio-Pléistocène dans la formation des reliefs des Pyrénées de l'Est doit être réévalué à la baisse. Au niveau de la charnière entre Conflent et Roussillon (bassin d'Ille-surTêt), le décalage de la surface repère calibrée (la transition marin-continental zancléenne) implique un rejet vertical de 150 à 250 m environ [8]. En Conflent, par soustraction de l'incision pléistocène à la hauteur des facettes actuelles, la part de la tectonique PlioPléistocène peut être évaluée à environ 200 m, soit un même ordre de grandeur. Ces valeurs paraissent représentatives de l'importance de la déformation pliocène. Quant au prolongement plus tardif de l'activité de la faille au cours du Pléistocène, envisagée par certains auteurs [4,7], sa part doit être réduite, voire nulle. Il n'existe en Roussillon aucune preuve directe de déformation pléistocène, les seules présomptions reposant sur la géométrie des terrasses anciennes [7,8]. La poursuite de la déformation au-delà du Pléistocène ancien est peu probable. En deux points du bassin du Conflent, la faille est en effet scellée par des cônes alluviaux enracinés sur le massif du Canigou. Ils sont attribués au Pléistocène moyen et leur géométrie exclut toute déformation. Ils constituent des terminus ante quem de la déformation et impliquent un régime tectonique faible à nul depuis cette époque en termes d'acquisition du relief.

Contrairement au modèle préalablement admis [7], il est donc improbable que l'essentiel du relief des Pyrénées de l'Est ait été acquis après le Miocène moyen. Nos propres résultats géomorphologiques, confirmant les données récentes obtenues par traces de fission sur apatite [19], suggèrent plutôt une formation ancienne du horst du Canigou à l'Oligocène (27$26 \mathrm{Ma}$ ), en relation avec l'ouverture du golfe du Lion. Cette formation s'accompagne de la mise en place d'un réseau hydrographique proche de l'actuel dans ses grands traits. Cet épisode est enregistré en Conflent par le dépôt des séries syn-tectoniques inférieures, datées de l'Oligo-Aquitanien [2]. Le rejet vertical lors de cet épisode a été évalué à 2000 m [22].

La question du moteur de l'incision pléistocène reste pour l'instant non débattue. L'étagement des formes auquel elle conduit ne saurait en effet résulter des seules variations du niveau de base glacioeustatique. Nos travaux antérieurs [9] et des travaux en cours (Rabineau, comm. pers.) suggèrent que ce moteur pourrait être à rechercher dans le basculement de la marge continentale au cours du Pléistocène, qui pourrait induire un réajustement asismique des reliefs bordiers.

\section{Remerciement}

Ce projet a bénéficié du soutien de l'Institut de radioprotection et de la sûreté nucléaire (IRSN).

\section{Références}

[1] S. Baize, E.M. Cushing, F. Lemeille, T. Granier, B. Grellet, D. Carbon, P. Combes, C. Hibsch, Sismotectonique de la France métropolitaine, vol. 3 : Inventaire des indices de rupture affectant le Quaternaire en relation avec les grandes structures connues, Mém. Soc. géol. France (2003) 175.

[2] Y. Bandet, Les terrains néogènes du Conflent et du Roussillon occidental, Thèse, Université Toulouse 3, 1975, 74 p.

[3] D. Bonnefoy, J. Chantraine, P. Lebret, D. Rabu, Carte géologique de la France à 1:1000000 numérique, Rapport BRGM R 39145, 1996.

[4] A. Briais, R. Armijo, T. Winter, P. Tapponnier, A. Herbecq, Morphological evidence for Quaternary normal faulting and 
seismic hazard in the Eastern Pyrenees, Ann. Tectonicae IV (1) (1990) 19-42.

[5] D.W. Burbank, R.S. Anderson, Tectonic Geomorphology, Blackwell Science, 2001, 274 p.

[6] L. Cabrera, E. Roca, P. Santanach, Basin formation at the end of a strike-slip fault: The Cerdanya Basin (eastern Pyrenees), J. Geol. Soc. 145 (1988) 261-268.

[7] M. Calvet, Morphogenèse d'une montagne méditerranéenne : Les Pyrénées orientales. Thèse, Université Paris 1, 1994, $1178 \mathrm{p}$.

[8] J.-M. Carozza, Évolution des systèmes géomorphologiques en contexte orogénique : l'Exemple des bassins d'alimentation des Pyrénées orientales. Approche morpho-tectonique. Thèse, Université Toulouse 2, 1998, 398 p.

[9] J.-M. Carozza, B. Delcaillau, L'enregistrement géomorphologique de la tectonique quaternaire par les nappes alluviales : l'Exemple du bassin de la Têt (Roussillon, France), C. R. Acad. Sci. Paris, Ser. IIa 329 (1999) 735-740

[10] J.-M. Carozza, B. Delcaillau, Réponse des bassins versants à l'activité tectonique : l'Exemple de la terminaison orientale de la chaîne pyrénéenne, Appr. Morphotecton. Géomorphol. 1 (2000) 45-60.

[11] J.-M. Carozza, S. Baize, B. Delcaillau, Incision and exhumation of the Têt Fault during the Pleistocene, Geodin. Acta (soumis).

[12] CEA/LDG, Catalogue de sismicité instrumentale 1962-1999, 2000

[13] G. Clauzon, Reconstitution de l'évolution géodynamique néogène du bassin du Roussillon et de l'unité adjacente des Corbières d'après les données éco-stratigraphiques et paléogéographiques, Paléobiologie Continentale XVII (1990) 125-155.

[14] G. Clauzon, S. Fauquette, J.-P. Suc, Quantification des paléo- altitudes néogènes des reliefs des Pyrénées orientales, in: Colloque du GdR Marges, 2002, pp. 40-41.

[15] P. Ferrer, E. Massana, P. Santanach, Expresion geomorfologica de la actividad reciente de la falla de Amer (NE de la Peninsula Iberica), Acta Geol. Hisp. 31 (4) (1996) 17-24.

[16] X. Goula, C. Olivera, J. Fleta, B. Grellet, R. Lindo, L.A. Rivera, A. Cisternas, D. Carbon, Present and recent stress regime in the eastern part of the Pyrenees, Tectonophysics 308 (1999) 487-502.

[17] G. Guitard, B. Laumonier, A. Autran, Y. Bandet, G.-M. Berger, Notice explicative, in : Carte géol. France $\left(1: 50000^{\mathrm{e}}\right)$, feuille de Prades (1095), Orléans, 1998, p. 198.

[18] B.U. Haq, J. Harenbol, P.R. Vail, Chronology of fluctuating sea levels since the Triassic, Science 235 (1987) 1156-1166.

[19] O. Maurel, M. Brunel, P. Monié, Exhumation cénozoïque des massifs du Canigou et de Mont-Louis (Pyrénées orientales, France), C. R. Geoscience 334 (2002) 941-948.

[20] H. Pauchet, Sismicité de la chaîne pyrénéenne : Image générale et analyse de la crise sismique de Saint-Paul-de-Fenouillet (février 1996). Implications tectoniques, Thèse, Université Toulouse 3, 1998, $155 \mathrm{p}$.

[21] H. Philip, J.-C. Bousquet, J. Escuer, J. Fleta, X. Goula, B. Grellet, Présence de failles inverses d'âge Quaternaire dans l'Est des Pyrénées : Implications sismotectoniques, C. R. Acad. Sci. Paris, Ser. II 314 (1992) 1239-1245.

[22] V. Sère, M. Brunel, J. Carpena, The uplift of the Canigou Massif (P.O., France): A fission tracks contribution, Terras Abstr., EUG, Terra Nova 7 (1995) 44.

[23] A. Souriau, M. Sylvander, A. Rigo, J.-F. Fels, J.-M. Douchain, C. Ponsolles, Sismotectonique des Pyrénées : Principales contraintes sismologiques, Bull Soc. géol. France 172 (2001) 25-40. 